\title{
Applications of the wave packet method to resonant transmission and reflection gratings
}

\author{
Andrei G. Borisov ${ }^{a, 1}$ and Sergei V. Shabanov ${ }^{b, 2}$ \\ ${ }^{a}$ Laboratory of Atomic and Molecular Collisions, University of Paris-Sud, Orsay, France \\ ${ }^{b}$ Department of Mathematics, University of Florida, Gainesville, FL 32611, USA
}

\begin{abstract}
Scattering of femtosecond laser pulses on resonant transmission and reflection gratings made of dispersive (Drude metals) and dielectric materials is studied by a time-domain numerical algorithm for Maxwell's theory of linear passive (dispersive and absorbing) media. The algorithm is based on the Hamiltonian formalism in the framework of which Maxwell's equations for passive media are shown to be equivalent to the first-order equation, $\partial \Psi / \partial t=\mathcal{H} \Psi$, where $\mathcal{H}$ is a linear differential operator (Hamiltonian) acting on a multi-dimensional vector $\Psi$ built of the electromagnetic inductions and auxiliary matter fields describing the medium response. The initial value problem is then solved by means of a modified time leapfrog method in combination with the Fourier pseudospectral method applied on a non-uniform grid that is constructed by a change of variables and designed to enhance the sampling efficiency near medium interfaces. The algorithm is shown to be highly accurate at relatively low computational costs. An excellent agreement with previous theoretical and experimental studies of the gratings is demonstrated by numerical simulations using our algorithm. In addition, our algorithm allows one to see real time dynamics of long leaving resonant excitations of electromagnetic fields in the gratings in the entire frequency range of the initial wide band wave packet as well as formation of the reflected and transmitted wave fronts.
\end{abstract}

\footnotetext{
${ }^{1}$ email: borisov@lcam.u-psud.fr

${ }^{2}$ email: shabanov@phys.ufl.edu
} 


\section{Introduction}

The purpose of the present study is twofold. First, we test a novel time-domain algorithm for the Maxwell's theory of linear, passive (dispersive and absorbing) media. The algorithm is based on $(i)$ the Hamiltonian formalism for evolution differential equations [1], on ( $i i)$ the time leapfrog scheme [2], and on (iii) the Fourier pseudospectral method [3] in combination with a change of variables that enhances the spatial grid resolution in designated domains (in the vicinity of medium interfaces) and, thereby, prevents the loss of accuracy due to the aliasing problem of the Fourier transform, while keeping the total spatial grid size fixed [4], [5]. Boundary conditions at medium interfaces are not fixed in the algorithm, but rather medium parameters are allowed to have spatial discontinuities so that the correct boundary conditions are enforced dynamically [6], similarly to the wave packet method for quantum mechanical systems with discontinuous potentials. Although, each of these three main ingredients of our algorithm have been used individually in various computational problems in electromagnetism and quantum mechanics, to our knowledge they have never been put together in applications to numerical simulations of propagation of a wideband electromagnetic pulse in passive media and its scattering on targets made of dispersive and absorbing materials. By combining these methods, we have obtained an efficient, true time-domain algorithm that is highly accurate, which is a known virtue of pseudospectral methods of solving partial differential equations [7].

An essential advantage of time-domain numerical methods is that one can see all the immediate effects the medium and targets have on the propagating wideband wave packet. Yet, a single simulation of scattering of a wideband wave packet is sufficient to determine some basic electromagnetic properties of a target, e.g., transmission and reflection coefficients, in the entire frequency range of the initial wave packet.

It has been reported [8] that a periodic thin-film metallic grating (either with holes or onedimensional slits) can transmit more light at certain wavelengths than the projected area of the holes (or slits) in the grating would suggest, while at other wavelengths transmission is almost fully blocked. There is an ongoing discussion about the mechanism of such anomalous transmission. Among suggested mechanisms are formation of dynamical diffraction resonances in periodic metallic structures [9], surface plasmons whose resonances are enhanced by the array of holes in the grating [10], and, specific to the slit gratings, open Fabry-Pérot resonant cavities [11]. Since all the approaches produce essentially identical predictions for the transmission and reflection coefficients (the far-field) of the slit grating, it is, perhaps, necessary to have a closer look at the details of the electromagnetic field dynamics in the vicinity of the grating where deviations of theoretical predictions of a particular mechanism from the actual exact solution of Maxwell's equations might occur (see, e.g., a discussion in a recent work [12]). This is our second motivation of the present study. We apply our algorithm to scattering of a wideband electromagnetic pulse on various transmission and reflection slit gratings (dispersive metallic and purely dielectric ones). Our time-domain algorithm allows one to observe in details (in real time) formation and decay of long-living resonant excitations of electromagnetic fields as well as formation of resonant transmission and reflection wave fronts. Thanks to the use of the Fourier 
pseudospectral method in combination with nonuniform grids, an extremely high accuracy of simulations can be achieved in the entire simulation volume and time span at relatively low computational costs. We think that these virtues of our time-domain algorithm would be useful for numerical studies of electromagnetic properties of other nanostructured materials [13].

The paper is organized as follows. Section 2 is devoted to the Hamiltonian formalism applied to the Maxwell's theory for general passive linear media. Maxwell's equations for electromagnetic fields and medium responses are shown to be equivalent to a first order evolution differential equation similar to the Schrödinger equation in quantum mechanics. The wave function is a multidimensional column whose components are electromagnetic fields and auxiliary fields describing the medium response. The Hamiltonian operator is a linear differential operator acting on a Hilbert space spanned by square integrable wave functions. In Section 3, an example of the multi-resonant Lorentz model, which is widely used to describe passive media, is considered in the framework of the Hamiltonian formalism. In Section 4, we establish a relation between the norm of wave functions and electromagnetic energy. The discussion is limited to the Lorentz model and non-dispersive dielectric media. Section 5 is devoted to a general description of our algorithm. In particular, we show how the action of the Hamiltonian on wave functions is defined on a grid by means of the fast Fourier transform method. We prove that the Gauss law is enforced in the grid representation at no extra computational costs in our algorithm. Then we discuss how the sampling efficiency of the fast Fourier method can be enhanced in designated spatial regions (typically at medium interfaces) by changing variables on the grid. The time evolution is done by a modified leapfrog method applied to the Schrödinger equation. We show that the conventional leapfrog method leads to an unstable algorithm for media with absorption and propose a general method to modify the leapfrog scheme to obtain a conditionally stable algorithm. Finally, we give an explicit realization of our algorithm in the case of the multi-resonant Lorentz model. Section 6 contains a detailed description of the actual computational scheme used in our simulations of extraordinary transmission and reflection grating. We analyze the stability of the scheme using our general approach developed in Section 5. Section 7 is devoted to our numerical results. We also compare them with previous theoretical and experimental studies. Section 8 contains a brief conclusion.

\section{Maxwell's theory for passive media in the Hamiltonian formalism}

Let $\mathbf{E}$ and $\mathbf{H}$ be electric and magnetic fields, respectively, $\mathbf{D}$ and $\mathbf{B}$ the corresponding inductions, and $\mathbf{P}$ and $\mathbf{M}$ the medium polarization and magnetization vectors. Boldface letters denote three-vector fields in $\mathbb{R}^{3}$. Propagation of an electromagnetic wave packet in passive linear media in the absence of external radiating sources is described by the following set of equations $[6]$

$$
\dot{\psi}^{I}(t)=\mathcal{H}_{0} \psi^{F}(t)
$$




$$
\begin{aligned}
\psi^{I}(t) & =\psi^{F}(t)+\psi^{R}(t)=\psi^{F}(t)+\int_{0}^{t} d \tau \chi(t-\tau) \psi^{F}(\tau), \\
\psi^{I} & =\left(\begin{array}{c}
\mathbf{D} \\
\mathbf{B}
\end{array}\right), \quad \psi^{F}=\left(\begin{array}{c}
\mathbf{E} \\
\mathbf{H}
\end{array}\right), \quad \psi^{R}=\left(\begin{array}{c}
\mathbf{P} \\
\mathbf{M}
\end{array}\right), \quad \mathcal{H}_{0}=\left(\begin{array}{cc}
0 & c \boldsymbol{\nabla} \times \\
-c \boldsymbol{\nabla} \times & 0
\end{array}\right),
\end{aligned}
$$

where $c$ is the speed of light in vacuum, the overdot denotes the partial derivative $\partial / \partial t$ with respect to time $t$, the spatial argument of the fields, denoted below by $\mathbf{r}$, is suppressed. For generic anisotropic media, the medium response function $\chi(t)$ is regarded as a linear operator (matrix) acting on the components of $\psi^{F}$. For isotropic media, it is a scalar. The response function is also position dependent for non-homogeneous media. Let $\psi^{I}(0)=\psi^{F}(0)$ be an initial wave packet with finite energy (finite $\mathbb{L}_{2}\left(\mathbb{R}^{3}\right)$ norm). We are interested in a finite norm solution of the initial value problem for Maxwell equations (2.1) subject to the constraint (the Gauss law)

$$
\boldsymbol{\nabla} \cdot \mathbf{B}(t)=\boldsymbol{\nabla} \cdot \mathbf{D}(t)=0
$$

The response function $\chi(t)$ is usually deduced from a microscopic model of the medium in question [6]. Therefore it is natural to assume that $\chi(t)$ is a fundamental solution of some linear evolution differential equation so that

$$
\mathcal{L}_{t} \psi^{R}(t)=\omega_{p} \psi^{F}(t)
$$

where $\mathcal{L}_{t}$ is a linear differential operator (a polynomial in $\partial / \partial t$ ) and $\omega_{p}$ describes a coupling between applied electromagnetic fields and matter. In general, $\omega_{g}$ is a position dependent matrix. Causality of the medium response requires that the Fourier transform $\tilde{\chi}(\omega)$ of the response function should have poles only in the lower part of the complex plane of $\omega$ [6] so that the Fourier transform of $(2.2)$ reads $\tilde{\psi}^{R}(\omega)=\tilde{\chi}(\omega) \tilde{\psi}^{F}(\omega)$. By taking the Fourier transform of Eq. (2.5), one finds that $\tilde{\mathcal{L}}(\omega) \tilde{\psi}^{R}(\omega)=\omega_{p} \tilde{\psi}^{F}(\omega)$ and, hence, $\mathcal{L}_{t}=\omega_{p}^{-1}[\tilde{\chi}(i \partial / \partial t)]^{-1}$. If the response function is known from measurements in the frequency domain, components of $[\tilde{\chi}(\omega)]^{-1}$ can always be approximated by a polynomial with sufficient accuracy in a frequency range of interest.

Now the Hamiltonian formalism [1] can be applied to (2.5) to transform it to an equivalent system of first-order differential equations

$$
\dot{\xi}(t)=\mathcal{H}_{M}^{F} \xi(t)+\mathcal{V}_{M F} \psi^{F}(t)
$$

where $\xi$ is a column of auxiliary fields which are linear combinations of the response field and its time derivatives, save for the one of the highest order. There exists a linear operator $\mathcal{R}$ such that $\psi^{R}(t)=\mathcal{R} \xi(t)$. Its explicit form depends on the details of going over to the Hamiltonian formalism. One can, for instance, identify the first component of $\xi$ with $\psi^{R}$. In this case, $\mathcal{R}$ projects the column $\xi$ onto its first component. The response function can be expressed through the operators $\mathcal{H}_{M}^{F}$ and $\mathcal{V}_{M F}$ and $\mathcal{R}$ by making use of the fundamental solution of Eq. (2.6)

$$
\chi(t)=\theta(t) \mathcal{R} e^{\mathcal{H}_{M}^{F} t} \mathcal{V}_{M F}
$$


where $\theta(t)$ is the Heaviside function. Equation (2.7) can be regarded as a condition on possible choices of the operators $\mathcal{H}_{M}^{F}$ and $\mathcal{V}_{M F}$ and $\mathcal{R}$.

Applying $\mathcal{R}$ to $(2.6)$ we find

$$
\dot{\psi}^{R}(t)=\mathcal{R H}_{M}^{F} \xi(t),
$$

where the relation $\mathcal{R} \mathcal{V}_{M F}=0$ has been used. It is not hard to be convinced that the latter relation holds when the first component of $\xi$ coincides with $\psi^{R}$. Any other choice of $\xi$ can be obtained by a canonical transformation [1] which is a linear nonsingular transformation of auxiliary fields,

$$
\xi \rightarrow \mathcal{S}_{M} \xi, \quad \operatorname{det} \mathcal{S}_{M} \neq 0 \text {. }
$$

According to (2.6) and (2.7), $\mathcal{R} \rightarrow \mathcal{R S}_{M}^{-1}, \mathcal{H}_{M}^{F} \rightarrow \mathcal{S}_{M} \mathcal{H}_{M}^{F} \mathcal{S}_{M}^{-1}$, and $\mathcal{V}_{M F} \rightarrow \mathcal{S}_{M} \mathcal{V}_{M F}$ and, therefore, the condition $\mathcal{R} \mathcal{V}_{M F}=0$ remains true in the new basis of auxiliary fields. Denoting $\mathcal{V}_{F M}=-\mathcal{R H}_{M}^{F}$ and substituting (2.2) and (2.8) into (2.1) Maxwell's evolution equations (2.1) can be written in the Hamiltonian form

$$
\dot{\psi}^{F}(t)=\mathcal{H}_{0} \psi^{F}(t)+\mathcal{V}_{F M} \xi(t)
$$

Finally, the electromagnetic and auxiliary fields are unified into one column (wave function) so that Eqs. (2.10) and (2.6) can be represented as a single first-order evolution equation

$$
\begin{aligned}
\dot{\Psi}^{F}(t) & =\mathcal{H}^{F} \Psi^{F}(t), \\
\Psi^{F} & =\left(\begin{array}{c}
\psi^{F} \\
\xi
\end{array}\right), \quad \mathcal{H}^{F}=\left(\begin{array}{cc}
\mathcal{H}_{0} & \mathcal{V}_{F M} \\
\mathcal{V}_{M F} & \mathcal{H}_{M}^{F}
\end{array}\right) .
\end{aligned}
$$

The index $F$ indicates that electromagnetic fields are used as independent electromagnetic degrees of freedom. We shall refer to (2.12) as to a field representation. Accordingly, an induction representation is obtained by the similarity transformation

$$
\Psi^{I}=\left(\begin{array}{c}
\psi^{I} \\
\xi
\end{array}\right)=\mathcal{S} \Psi^{F}, \quad \mathcal{S}=\left(\begin{array}{cc}
1 & \mathcal{R} \\
0 & 1
\end{array}\right), \quad \mathcal{H}^{I}=\mathcal{S H}^{F} \mathcal{S}^{-1}
$$

The corresponding blocks of $\mathcal{H}^{I}$ have the form

$$
\begin{aligned}
\mathcal{H}_{I} & =\mathcal{H}_{0}, \quad \mathcal{V}_{M I}=\mathcal{V}_{M F}, \\
\mathcal{V}_{I M} & =\mathcal{V}_{F M}+\mathcal{R H}_{M}^{F}-\mathcal{H}_{0} \mathcal{R}=-\mathcal{H}_{0} \mathcal{R} \\
\mathcal{H}_{M}^{I} & =\mathcal{H}_{M}^{F}-\mathcal{V}_{M F} \mathcal{R} .
\end{aligned}
$$

In what follows we denote wave functions by $\Psi^{Q}(t)$ with $Q$ being the representation index, $F$ or $I$. This completes construction of the Hamiltonian representation of Maxwell's theory for linear passive media.

Boundary conditions at medium interfaces and possible scatterers (targets) are not imposed on electromagnetic fields, but rather they are enforced dynamically by allowing medium parameters to be discontinuous functions. The fundamental solution of (2.11)

$$
\Psi^{F}(t)=e^{t \mathcal{H}^{F}} \Psi^{F}(0), \quad t \geq 0
$$


is well defined for discontinuous "potentials" $\mathcal{V}_{M F}$ and $\mathcal{V}_{F M}$, for example, by means of the Kato-Trotter product formula used in the path integral representation of (2.17) as shown in [14].

\section{The Lorentz model}

The Hamiltonian formalism for the Lorentz model has been used in [15] to develop a finite differencing algorithm to study an electromagnetic pulse propagation in Lorentz media. Here we derive an explicit form of the Hamiltonian for the Lorentz model which is used in Section 5 for the stability analysis of our algorithm. The Lorentz model of a passive medium is based on the assumption that the medium magnetization is zero, $\mathbf{M}=0$, while the medium polarization is described by a set of decoupled second-order differential equations [6]

$$
\ddot{\mathbf{P}}_{a}+2 \gamma_{a} \dot{\mathbf{P}}_{a}+\omega_{a}^{2} \mathbf{P}_{a}=\omega_{p a}^{2} \mathbf{E}, \quad \mathbf{P}=\sum_{a=1}^{N} \mathbf{P}_{a}
$$

where $\omega_{a}$ are resonant frequencies, $\gamma_{a}$ are damping coefficients, and $\omega_{p a}$ are plasma frequencies. As has been pointed out, no boundary conditions are imposed on electromagnetic fields at medium and/or target interfaces. Instead, the coupling constants $\omega_{p a}=\omega_{p a}(\mathbf{r})$ are allowed to have discontinuities at medium interfaces, or, from the physical point of view, they remain smooth but change rapidly, $\lambda_{w}\left|\nabla \omega_{p}\right| / \omega_{p} \gg 1$, at the interface, where $\lambda_{w}$ is a typical wave length of the incident wave packet. The initial value problem is solved in the space of square integrable wave functions. Initial conditions for the response field are $\mathbf{P}(t=0)=\dot{\mathbf{P}}(t=0)=0$.

Consider $2 N$ real vector fields, $\boldsymbol{\xi}_{j}, j=1,2, \ldots, 2 N$, such that

$$
\begin{aligned}
\mathbf{P}_{a} & =\left(\omega_{p a} / \omega_{a}\right) \boldsymbol{\xi}_{2 a-1}, \\
\dot{\boldsymbol{\xi}}_{2 a-1} & =\omega_{a} \boldsymbol{\xi}_{2 a}, \quad \dot{\boldsymbol{\xi}}_{2 a}=-2 \gamma_{a} \boldsymbol{\xi}_{2 a}-\omega_{a} \boldsymbol{\xi}_{2 a-1}+\omega_{p a} \mathbf{E} .
\end{aligned}
$$

Thus, the original system of second order equations has been converted into the first order system. The operator $\mathcal{R}$ is defined by (3.2). After simple algebraic transformations, we infer

$$
\begin{aligned}
\mathcal{V}_{F M} & =\left(\mathcal{V}_{F M 1}, \mathcal{V}_{F M 2}, \cdots, \mathcal{V}_{F M N}\right), \quad \mathcal{V}_{F M a}=\left(\begin{array}{cc}
0 & -\omega_{p a} \\
0 & 0
\end{array}\right), \\
\mathcal{V}_{M F} & =-\mathcal{V}_{F M}^{\dagger}, \\
\mathcal{H}_{M}^{F} & =\operatorname{diag}\left(\mathcal{H}_{M 1}^{F}, \mathcal{H}_{M 2}^{F}, \cdots, \mathcal{H}_{M N}^{F}\right), \quad \mathcal{H}_{M a}^{F}=\left(\begin{array}{cc}
0 & \omega_{a} \\
-\omega_{a} & -2 \gamma_{a}
\end{array}\right),
\end{aligned}
$$

where diag indicates that the corresponding matrix is block-diagonal with blocks listed in the order from the upper left to lower right corners. Note that the matrices $\mathcal{V}_{F M a}$ and $\mathcal{H}_{F M a}$ act on a six-dimensional column $\xi_{a}$ composed of two vectors $\boldsymbol{\xi}_{2 a-1}$ and $\boldsymbol{\xi}_{2 a}$. Therefore they should 
be understood as composed of $3 \times 3$ blocks. Each block is obtained by multiplying the unit matrix by the number indicated in place of the block in (3.4) and (3.6).

Another convenient way to introduce the Hamiltonian formalism is to use $N$ complex vector fields $\boldsymbol{\zeta}_{a}$ which satisfy the first order differential equation

$$
\dot{\boldsymbol{\zeta}}_{a}=\lambda_{a} \boldsymbol{\zeta}_{a}-i \omega_{p a} \mathbf{E}, \quad \mathbf{P}_{a}=\frac{\omega_{p a}}{2 \nu_{a}}\left(\boldsymbol{\zeta}_{a}+\overline{\boldsymbol{\zeta}}_{a}\right)
$$

where $\lambda_{a}=-\gamma_{a}+i \nu_{a}$ and $\nu_{a}=\sqrt{\omega_{a}^{2}-\gamma_{a}^{2}}$. This representation is defined only if $\gamma_{a}<\omega_{a}$ (i.e., the attenuation is not high). From the numerical point of view, solving a decoupled system of $N$ first order differential equation and taking complex conjugation (denoted here by an over bar) is less expensive than solving the original system of second-order differential equations for the medium polarization. In general, there is always a freedom of choosing a new basis for the auxiliary field space (2.9). If the evolution operator $\exp \left(t \mathcal{H}^{Q}\right)$ is computed in one basis, it can be computed in another basis by a suitable similarity transformation. This is an important observation because the auxiliary field basis can be chosen in a way that facilitates computation of the evolution operator, e.g., to speed up simulations. For instance, in the complex representation (3.7), the matter Hamiltonian $\mathcal{H}_{M}^{F}$ is diagonal. The corresponding transformation of auxiliary fields is given by

$$
\left(\begin{array}{c}
\boldsymbol{\xi}_{2 a-1} \\
\boldsymbol{\xi}_{2 a}
\end{array}\right)=\frac{1}{2 \nu_{a}}\left(\begin{array}{cc}
\omega_{a} & \omega_{a} \\
\lambda_{a} & \bar{\lambda}_{a}
\end{array}\right)\left(\begin{array}{c}
\boldsymbol{\zeta}_{a} \\
\overline{\boldsymbol{\zeta}}_{a}
\end{array}\right) \equiv \mathcal{S}_{M}\left(\begin{array}{c}
\boldsymbol{\zeta}_{a} \\
\overline{\boldsymbol{\zeta}}_{a}
\end{array}\right)
$$

To transform the whole system into this representation, the Hamiltonian $\mathcal{H}^{F}$ is replaced by $\mathcal{S}^{-1} \mathcal{H}^{F} \mathcal{S}$ and the wave function $\Psi^{F}$ by $\mathcal{S} \Psi^{F}$ where $\mathcal{S}$ is block-diagonal with the unit matrix in the upper left (field) corner and with $\mathcal{S}_{M}$ in the lower right (matter) corner.

\section{Energy and the norm of state vectors}

Accuracy and convergence of a numerical algorithm is defined relative to some norm. Let us discuss the choice of a norm in the space spanned by wave functions $\Psi^{Q}(t)$. The discussion is limited to the Lorentz model and the case of a nonhomogeneous, nondispersive medium (dielectric) which are used in our numerical simulations.

\section{The Lorentz model}

Consider a multi-resonant Lorentz model with no attenuation $\gamma_{a}=0$. The field and matter evolution equations can be obtained from the variational principle for the action

$$
S=\int d t L=\int d t \int d \mathbf{r}\left[\frac{1}{2}\left(\mathbf{E}^{2}-\mathbf{B}^{2}\right)+\frac{1}{2} \sum_{a}\left(\dot{\boldsymbol{\vartheta}}_{a}^{2}-\omega_{a}^{2} \boldsymbol{\vartheta}_{a}^{2}\right)+\mathbf{P} \cdot \mathbf{E}\right]
$$


where the polarization of the medium is expressed via matter fields as $\mathbf{P}=\sum_{a} \omega_{p a} \boldsymbol{\vartheta}_{a}$. The electromagnetic degrees of freedom are described by the vector and scalar potentials, respectively, $\mathbf{A}$ and $\varphi$. The fields are defined by $\mathbf{E}=-\boldsymbol{\nabla} \varphi-\dot{\mathbf{A}}$ and $\mathbf{B}=\boldsymbol{\nabla} \times \mathbf{A}$. The units are chosen in this Section so that $c=1$. The least action principle for the scalar potential $\varphi$ leads to the Gauss law, $\boldsymbol{\nabla} \cdot \mathbf{D}=0$, for the vector potential $\mathbf{A}$ to the Maxwell's equation, $\dot{\mathbf{D}}=\boldsymbol{\nabla} \times \mathbf{B}$, and for the matter fields $\boldsymbol{\vartheta}_{a}$ to the medium polarization evolution equation (3.1) of the Lorentz model with no attenuation, $\gamma_{a}=0$. The second Maxwell's equation and the Gauss law for the magnetic field follows from the relation $\mathbf{B}=\boldsymbol{\nabla} \times \mathbf{A}$ by taking its time derivative and divergence, respectively.

The energy of the system coincides with the canonical Hamiltonian which is obtained by the Legendre transformation [1] of the Lagrangian $L$ for the velocities $\dot{\mathbf{A}}$ and $\dot{\boldsymbol{\vartheta}}_{a}$. The canonical momenta are $\boldsymbol{\pi}_{a}=\delta L / \delta \dot{\boldsymbol{\vartheta}}_{a}=\dot{\boldsymbol{\vartheta}}_{a}$ and $\boldsymbol{\Pi}=\delta L / \delta \dot{\mathbf{A}}=-\mathbf{E}-\mathbf{P}=-\mathbf{D}$. Doing the Legendre transformation, we find the canonical Hamiltonian (energy) of the system

$$
E(t)=\int d \mathbf{r}\left(\sum_{a} \boldsymbol{\pi}_{a} \cdot \dot{\boldsymbol{\vartheta}}_{a}+\mathbf{\Pi} \cdot \dot{\mathbf{A}}\right)-L=\frac{1}{2} \int d \mathbf{r}\left[\mathbf{E}^{2}+\mathbf{B}^{2}+\sum_{a}\left(\boldsymbol{\pi}_{a}^{2}+\omega_{a}^{2} \boldsymbol{\vartheta}_{a}^{2}\right)\right]
$$

where the Gauss law $\boldsymbol{\nabla} \cdot \mathbf{D}=0$ has been used. The energy conservation follows directly from the Noether theorem [1] applied to the time translation symmetry of the action $(4.1), \dot{E}(t)=0$. Equation (4.2) becomes the conventional expression for the electromagnetic energy in a passive medium [6] when $\boldsymbol{\pi}_{a}$ and $\boldsymbol{\vartheta}_{a}$ are replaced by the corresponding solutions of the equations of motion with initial conditions $\boldsymbol{\pi}_{a}(t=0)=\boldsymbol{\vartheta}_{a}(t=0)=0$.

An important observation is that the Noether integral of motion (4.2) coincides with the norm squared of the corresponding state vector

$$
E=\frac{1}{2} \int d \mathbf{r} \Psi^{F \dagger} \Psi^{F} \equiv\left(\Psi^{F}, \Psi^{F}\right)=\left(\Psi^{I}, \mu \Psi^{I}\right)
$$

where $\mu=\mathcal{S}^{-1 \dagger} \mathcal{S}^{-1}$. This follows from the fact that, if we identify $\boldsymbol{\xi}_{2 a}=\boldsymbol{\pi}_{a}$ and $\boldsymbol{\xi}_{2 a-1}=\omega_{a} \boldsymbol{\vartheta}_{a}$, the Hamiltonian equations of motion, $\dot{\boldsymbol{\vartheta}}_{a}=\delta E / \delta \boldsymbol{\pi}_{a}$ and $\dot{\boldsymbol{\pi}}_{a}=-\delta E / \delta \boldsymbol{\vartheta}_{a}$, coincide with (3.3) when $\gamma_{a}=0$. Note that canonically conjugated electromagnetic variables are $\mathbf{A}$ and $-\mathbf{D}$. Therefore, the coupling between the electromagnetic and matter degrees of freedom in the Hamiltonian equations of motion is generated by the term $\mathbf{E}^{2}=(\mathbf{D}-\mathbf{P})^{2}$ in (4.2). Thus, in the absence of attenuation, the norm of the state vector is proportional to the wave packet electromagnetic energy and, hence, is conserved.

The norm conservation also follows from anti-Hermiticity of the Hamiltonian $\mathcal{H}^{F \dagger}=-\mathcal{H}^{F}$ if $\gamma_{a}=0$, while (4.3) establishes a relation between the electromagnetic energy and the norm. In the induction representation, the norm in the measure space, defined by the operator $\mu$ in (4.3), is also conserved by construction. Consequently, the Hamiltonian is anti-Hermitian relative to the measure space scalar product, $\mathcal{H}^{I \dagger} \mu=-\mu \mathcal{H}^{I}$.

The norm (energy) conservation can be used to control numerical convergence, especially when the aliasing problem in the fast Fourier transform is present, e.g., when parameters of 
the medium are discontinuous functions in space. In a properly designed algorithm the loss of energy (norm) due to attenuation should be controlled by the symmetric part of the Hamiltonian operator

$$
\dot{E}(t)=-\sum_{a} \gamma_{a} \int d \mathbf{r} \boldsymbol{\xi}_{2 a}^{2}(t) \equiv \frac{1}{2}\left(\Psi^{F}(t), \mathcal{V}_{\gamma}^{F} \Psi^{F}(t)\right) \leq 0,
$$

where $\mathcal{V}_{\gamma}^{F \dagger}=\mathcal{V}_{\gamma}^{F}=\left(\mathcal{H}^{F \dagger}+\mathcal{H}^{F}\right) / 2 \leq 0$ (a negative semidefinite operator) which is, in this case, a diagonal matrix with nonpositive elements.

\section{Nondispersive media}

If the medium in question does not have dispersion and absorption, the formalism is simplified. Let $\varepsilon=\varepsilon(\mathbf{x})$ be the dielectric constant of the medium, $\mathbf{D}=\varepsilon \mathbf{E}$. If the medium is not isotropic, then $\varepsilon$ is symmetric positive definite $3 \times 3$ matrix everywhere in space. We rewrite Maxwell's equations in the form

$$
\dot{\psi}^{I}(t)=\mathcal{H}_{G} \psi^{I}(t), \quad \mathcal{H}_{G}=\left(\begin{array}{cc}
0 & c \boldsymbol{\nabla} \times \\
-c \boldsymbol{\nabla} \times\left(\varepsilon^{-1}\right) & 0
\end{array}\right),
$$

where the parenthesis in $\left(\varepsilon^{-1}\right)$ mean that the induction is first multiplied by $\varepsilon^{-1}$ and then the curl of the resulting vector field is computed. Consider the scalar product

$$
\left(\psi_{1}^{I}, \mu_{\varepsilon} \psi_{2}^{I}\right)=\int d \mathbf{r} \psi_{1}^{I \dagger} \mu_{\varepsilon} \psi_{2}^{I}, \quad \mu_{\varepsilon}=\left(\begin{array}{cc}
\varepsilon^{-1} & 0 \\
0 & 1
\end{array}\right) .
$$

The Hamiltonian is anti-Hermitian with respect to this measure space scalar product, $\mathcal{H}_{G}^{\dagger} \mu_{\varepsilon}=$ $-\mu_{\varepsilon} \mathcal{H}_{G}$. Therefore the corresponding norm is preserved in the time evolution generated by $\exp \left(t \mathcal{H}_{G}\right)$, that is, $\left(\psi^{I}(t), \mu_{\varepsilon} \psi^{I}(t)\right)=\left(\psi^{I}(0), \mu_{\varepsilon} \psi^{I}(0)\right)$. The electromagnetic energy of the wave packet is conserved because it is proportional to the measure space norm of the initial state vector.

\section{The algorithm}

\section{The grid representation}

Consider an equidistantly spaced finite grid with periodic boundary conditions. Let $\Delta r$ be the grid step and $\mathbf{n}$ be a vector with integer valued components. Then the dynamical variables are projected onto the grid by taking their values at grid points $\mathbf{r}=\mathbf{n} \Delta r$, that is, the wave function is replaced by a vector (column) whose dimension is determined by the grid size, $\Psi^{Q}(\mathbf{r}) \rightarrow \Psi^{Q}(\mathbf{n} \Delta r) \equiv \Psi_{\mathbf{n}}^{Q}$. A cubic grid is assumed. Consider a discrete Fourier transformation associated with the grid, $\tilde{\Psi}^{Q}\left(\mathbf{n} k_{0}\right)=\sum_{\mathbf{n}^{\prime}} \mathcal{F}_{\mathbf{n n}^{\prime}} \Psi_{\mathbf{n}^{\prime}}^{Q} \equiv \tilde{\Psi}_{\mathbf{n}}^{Q}$, where $\mathcal{F}^{\dagger} \mathcal{F}=\mathcal{F}^{\dagger}=1$. The 
reciprocal lattice step is $k_{0}=2 \pi / \Delta r$. The grid spatial size $L$ and step $\Delta r$ must be chosen so that the Fourier transform of the initial wave packet has support within the region $k \in\left[k_{\min }, k_{\text {max }}\right]$ where $k=|\mathbf{k}|, k_{\max }=k_{0}$ and $k_{\min }=2 \pi / L$. The action is of any position dependent operator $\mathcal{V}^{Q}=\mathcal{V}^{Q}(\mathbf{r})$ is defined by

$$
\mathcal{V}^{Q}(\mathbf{r}) \Psi_{t}^{Q}(\mathbf{r}) \rightarrow \mathcal{V}^{Q}(\mathbf{n} \Delta r) \Psi_{t}^{Q}(\mathbf{n} \Delta r) \equiv \sum_{\mathbf{n}^{\prime}} \mathcal{V}_{\mathbf{n n}^{\prime}}^{Q} \Psi_{\mathbf{n}^{\prime}}^{Q}
$$

where $\mathcal{V}_{\mathbf{n n}^{\prime}}^{Q}=\delta_{\mathbf{n n}^{\prime}} \mathcal{V}^{Q}(\mathbf{n} \Delta r)$ is a diagonal matrix. Let $\mathcal{H}_{0}^{Q}=\mathcal{H}_{0}^{Q}(\boldsymbol{\nabla})$ depend only on the $\boldsymbol{\nabla}$ operator. The projection of its action onto the grid is then defined via the discrete Fourier transform

$$
\left.\mathcal{H}_{0}^{Q}(\boldsymbol{\nabla}) \Psi_{t}^{Q}(\mathbf{r})\right|_{\mathbf{r}=\mathbf{n} \Delta r} \rightarrow \sum_{\mathbf{n}^{\prime}} \mathcal{H}_{0 \mathbf{n n}^{\prime}}^{Q} \Psi_{\mathbf{n}^{\prime}}^{Q}, \quad \mathcal{H}_{0 \mathbf{n} \mathbf{n}^{\prime}}^{Q}=\sum_{\mathbf{n}^{\prime \prime}}\left(\mathcal{F}^{\dagger}\right)_{\mathbf{n n}^{\prime \prime}} \mathcal{H}_{0}^{Q}\left(i \mathbf{n}^{\prime \prime} k_{0}\right)(\mathcal{F})_{\mathbf{n}^{\prime \prime} \mathbf{n}^{\prime}}
$$

The projection (5.2) is performed by the fast Fourier transform method.

In what follows, the action of a product of $\mathcal{V}^{Q}$ and $\mathcal{H}_{0}^{Q}$ on any state vector is understood as multiplication of $\Psi_{\mathbf{n}}^{Q}$ by the corresponding matrices, defined in (5.1) and (5.2), in the order specified in the product. The main advantage of using the Fourier basis is the exponential convergence (versus the polynomial one in finite differencing schemes) [7] as the grid size increases, which allows one to substantially increase the accuracy of simulations.

\section{The Gauss law}

Another advantage of the Fourier basis is that the Gauss law is enforced at no extra computational cost. In the grid representation defined above, the Gauss law (2.4) requires that the Fourier transforms of the inductions $\tilde{\mathbf{D}}(\mathbf{k})$ and $\tilde{\mathbf{B}}(\mathbf{k})$ remains perpendicular to the reciprocal grid vector $\mathbf{k}=\mathbf{n} k_{0}$ at any moment of time. In our algorithm, as we shall show shortly, the time evolution is generated by applying powers of the Hamiltonian to the wave function. In the induction representation, the action of powers of $\mathcal{H}^{I}$ always produces the cross product $\mathbf{k} \times \tilde{\mathbf{C}}(\mathbf{k})$, for some $\tilde{\mathbf{C}}(\mathbf{k})$ regular at $\mathbf{k}=0$, in the entries of $\Psi^{I}$ that correspond to the electromagnetic inductions. Hence, in the grid representation the wave function $\left(\mathcal{H}^{I}\right)^{m} \Psi^{I}$ satisfies the Gauss law for any power $m$ because of the trivial identity $\mathbf{k} \cdot(\mathbf{k} \times \tilde{\mathbf{C}}(\mathbf{k}))=0$ valid for any vector $\mathbf{k}$ of the reciprocal grid.

It should be noted that a high accuracy of the Gauss law is essential to achieve a high accuracy of simulated electromagnetic fields near medium interfaces.

\section{Improving sampling efficiency by changing variables}

As is well known from the Fourier analysis, the convergence rate can be affected for functions which have discontinuities [3]. The latter is, unfortunately, the case in electromagnetic scattering problems [6]. Suppose there is an interface between two media. It can be deduced from 
the dynamical Maxwell's equations that the components of electric and magnetic fields, E and $\mathbf{H}$, tangential to the interface must be continuous, provided there is no surface electric current on the interface. From the Gauss law it follows that components of the inductions, D and B, normal to the interface must be continuous, provided there is no surface charge on the interface. In contrast, normal components of the fields and tangential components of the inductions can be discontinuous. Their discontinuities are determined by discontinuities of medium parameters (e.g., discontinuities in plasma frequencies in Lorentz models). Therefore, in either the induction or field representation, there are components which suffer discontinuities at the interface. The only way to cope with the problem, while keeping the use of the Fourier basis, is to make the grid finer [3]. This would lead to a substantial waste of computational resources because the conventional fast Fourier transform is defined on a uniform periodic grid, while the sampling efficiency should only be enhanced in the neighborhood of medium interfaces. The use of wavelet bases might be helpful for such a task [16] in time domain algorithms. Here we retain the Fourier basis, and increase the sampling efficiency by a change of variables.

The basic idea can be understood with a one-dimensional example. Let $z$ be a physical coordinate. Consider a change of variables defined by $z=f(y)$ where $y$ is an auxiliary coordinate. An equidistant grid $y_{n}=n \Delta y$, with $n$ being integers, of the auxiliary coordinate generates a non-uniform grid of the the physical coordinate, $z_{n}=f(n \Delta y)$. Assuming $\Delta y$ to be sufficiently small and $f(y)$ sufficiently smooth, the physical grid spacing can be approximated as

$$
\Delta z_{n}=z_{n+1}-z_{n} \approx \Delta y f^{\prime}(n \Delta y) .
$$

So, if $f^{\prime}(y)=1$, then $\Delta z_{n}=\Delta y$ and the grid is equidistant. By making the derivative $0<f^{\prime}(y) \leq 1$ in some designated areas, one can achieve a desired local grid density in the physical space, while keeping the total grid size fixed. For example, if it is necessary to increase the sampling efficiency in the vicinity $z=0$, one can take $f^{\prime}(y)=1-a_{0}\left[1+b_{0}^{2} y^{2}\right]^{-1}$, where $0<a_{0}<1$, and, hence,

$$
f(y)=y-\frac{a}{b} \tan ^{-1}(b y) \equiv y-g(y, a, b) .
$$

By adjusting parameters $a$ and $b$, the local grid density can be changed as desired. Consequently, if the sampling efficiency is to be enhanced at several points $y_{i}$, a suitable change of variables can be of the form $f(y)=y-\sum_{i} g\left(y-y_{i}, a_{i}, b_{i}\right)$. The fast Fourier transform is applied on a uniform grid of the auxiliary variable.

Upon the change of variables, the integration measure in the scalar product and the derivative transform, respectively, $d z=d y f^{\prime}(y)$ and $\partial_{z}=\left[f^{\prime}(y)\right]^{-1} \partial_{y}$. When projected on a uniform grid of the physical coordinate by means of (5.2), the derivative operator $\partial_{z}$ becomes an antiHermitian matrix. When the rule (5.2) is applied on a uniform grid of the auxiliary coordinate $y$, the derivative operator $\partial_{z}$ is no longer represented by an anti-Hermitian matrix, although it is still an anti-Hermitian linear operator, but in the measure space where the scalar product is defined with the weight $f^{\prime}(n \Delta y)$ at each lattice cite. From the numerical point of view it is convenient to have an explicitly anti-Hermitian matrix representation of the derivative on the grid. Due to round-off errors, the exact anti-Hermiticity of $\partial_{z}$ in the measure space can 
be violated in the grid representation, which, in turn, may lead to numerical instabilities of simulations. Note that if a computed matrix is known to be anti-Hermitian, then only half of its elements is to be computed, while the other elements are restored by the symmetry. In the measure space, an explicit anti-Hermiticity of a linear operator is much more difficult to maintain in numerical simulations because the symmetry relation between matrix elements depends on the scalar product measure. For this reason, the wave function is rescaled by the square root of the Jacobian [5], $\Psi \rightarrow \sqrt{f^{\prime}} \Psi$ so that the integration measure becomes $d y$ leading to the conventional Euclidean scalar product in the grid representation (with the uniform unit weight at each grid site). In such a representation, the derivative operator $\partial_{z} \rightarrow\left[f^{\prime}\right]^{-1 / 2} \partial_{y}\left[f^{\prime}\right]^{-1 / 2}$ becomes again an explicitly anti-Hermitian matrix in the grid representation defined by the rules (5.1) and (5.2).

\section{A modified temporal leapfrog scheme}

A conventional temporal leapfrog method of solving the Schrödinger equation is based on the iteration scheme [2]

$$
\Psi^{Q}(t+\Delta t)=\Psi^{Q}(t-\Delta t)+2 \Delta t \mathcal{H}^{Q} \Psi^{Q}(t)
$$

so that the wave function in the consecutive time moment is computed from the wave function at two previous moments of time, where $\Delta t$ is the time step. The action of the Hamiltonian $\mathcal{H}^{Q}$ is computed by pseudospectral methods, in particular, by means of the Fourier basis and the fast Fourier transform in our algorithm. The leapfrog algorithm is conditionally stable for an anti-Hermitian $\mathcal{H}^{Q}$, which is true for nondispersive media, but not the case for media with absorption.

To investigate the stability, let us introduce the amplification matrix for the leapfrog algorithm, $\Psi(t+\Delta t)=\mathcal{G}(\Delta t) \Psi(t)$ (the representation index, $Q$, is suppressed for a moment). From (5.3) it follows that $\mathcal{G}(\Delta t)$ satisfies the equation $\mathcal{G}^{2}(\Delta t)-2 \Delta t \mathcal{H} \mathcal{G}(\Delta t)-1=0$ which has two solutions

$$
\mathcal{G}^{( \pm)}(\Delta t)=\mathcal{H} \Delta t \pm \sqrt{1+\mathcal{H}^{2} \Delta t^{2}}
$$

The stability of the algorithm requires that the energy norm of both approximate solutions $\left[\mathcal{G}^{( \pm)}(\Delta t)\right]^{n} \Psi(0)$ must be uniformly bounded for $n>0$. The necessary condition (but not sufficient) is the von Neumann condition that the spectral radius of the amplification matrix does not exceed 1. If a complex number $R e^{i \varphi}$ is an eigenvalue of $\Delta t \mathcal{H}$, then the von Neumann condition implies that $\varphi= \pm \pi / 2$ and $R^{2} \leq 1$. In other words, eigenvalues of $\Delta t \mathcal{H}$ must be imaginary and their magnitude should not exceed 1. If in addition we demand that the Hamiltonian is diagonalizable, then a conditional stability can be achieved for sufficiently small $\Delta t$. Indeed, in this case there exists an non-singular $\mathcal{S}$ such that

$$
\mathcal{H}=\mathcal{S}^{-1} \mathcal{H}_{S} \mathcal{S}, \quad \mathcal{H}_{S}^{\dagger}=-\mathcal{H}_{S}
$$

and $\Delta t \mathcal{H}_{S}$ satisfies the von Neumann condition. Since $\mathcal{H}$ and $\mathcal{H}_{S}$ have the same eigenvalues, the amplification matrices for $\mathcal{H}$ and $\mathcal{H}_{S}$ are related by the same similarity transformation (5.5). 
Hence, the norm of a wave function obtained by the action of powers of (5.4) on an initial wave function is uniformly bounded. Note also that the Hamiltonian (5.5) is anti-Hermitian relative to the measure space scalar product, $\mathcal{H}^{\dagger} \mu=-\mu \mathcal{H}$, where $\mu=\mathcal{S}^{-1 \dagger} \mathcal{S}^{-1}$. The stability can also be proved via the equivalence of the conventional Euclidean norm and the $\mu$-norm [14].

In the case of nondispersive media, the Hamiltonian $\mathcal{H}_{G}$ is anti-Hermitian in the measure space, and the von Neumann condition is fulfilled if

$$
\Delta t c k_{\max }^{\varepsilon} \leq 1
$$

where $k_{\max }^{\varepsilon}$ the maximal norm of all wave vectors in the medium which can be estimated by $\sqrt{\rho(\varepsilon)} k_{\max }$ with $k_{\max }$ being the maximal wave vector of the initial pulse in vacuum and $\rho(\varepsilon)$ the maximal spectral radius of the symmetric matrix $\varepsilon(\mathbf{x})$ over $\mathbf{x}$ (or simply the maximum of $\varepsilon(\mathbf{x})$ if the medium is isotropic). This can be understood from the following principle [17]. A finite difference scheme with variable coefficients is stable if all the corresponding schemes with frozen (i.e., fixed to a particular value everywhere in space) coefficients are stable.

The von Neumann condition cannot be met if absorption is present because eigenvalues of the Hamiltonian must have real parts in order to account for exponential attenuation of field amplitudes. To circumvent this difficulty, the leapfrog scheme is modified in the following way [14]. We assume the Hamiltonian to be diagonalizable. The lack of eigenvectors of the Hamiltonian typically leads to solutions whose amplitudes grow polynomially in time [14]. This feature cannot be present in a physically reasonable model of passive media. So our assumption is justified from the physical point of view and, yet, the Lorentz model Hamiltonian is indeed diagonalizable. Let

$$
\mathcal{H}=\mathcal{H}_{0}+\mathcal{V}, \quad(\Psi, \mathcal{V} \Psi) \leq 0
$$

for any $\Psi$ in the Hilbert space and the von Neumann condition is satisfied for $\mathcal{H}_{0}$, i.e., $\mathcal{H}_{0}$ has imaginary eigenvalues. The split (5.7) can be achieved in many ways. For instance, $\mathcal{H}_{0}$ can be obtained from $\mathcal{H}$ by setting all parameters responsible for attenuation to zero. In a physically acceptable model, the energy must be conservative in an absorption free medium and so must be the energy norm of wave vectors, and, therefore, the corresponding Hamiltonian must be anti-Hermitian (relative to the energy induced scalar product). In the Lorentz model this is easily seen in the field representation if we set $\mathcal{H}_{0}=\left.\mathcal{H}\right|_{\gamma_{a}=0}$ which is explicitly anti-Hermitian (see Section 3). Then $\mathcal{V}$ is diagonal with matrix elements being zeros and $-2 \gamma_{a}$. Another possibility is to identify $\mathcal{H}_{0}$ with the Hamiltonian in the vacuum, then $\mathcal{V}=\mathcal{H}-\mathcal{H}_{0}$ must be negative semidefinite in order to model exponential attenuation in passive media. Finally, one can also split the Hamiltonian into the sum of Hermitian and anti-Hermitian parts.

After choosing a suitable split (5.7) we make a substitution $\Psi(t)=\exp (t \mathcal{V}) \Phi(t)$ in the original evolution equation (2.11). The new wave function $\Phi(t)$ satisfies the equation with a time dependent Hamiltonian

$$
\dot{\Phi}(t)=e^{-t \mathcal{V}} \mathcal{H}_{0} e^{t \mathcal{V}} \Phi(t) \equiv \mathcal{H}(t) \Phi(t),
$$

to be solved with the same initial condition $\Phi(0)=\Psi(0)$. Applying the leapfrog method to (5.8) we get $\Phi(t+\Delta t)=\Phi(t-\Delta t)+2 \Delta t \mathcal{H}(t) \Phi(t)$ valid up to $O\left(\Delta t^{3}\right)$. Returning to the initial 
variables, we arrive at the following recurrence relation

$$
\Psi(t+\Delta t)=\mathcal{L}(2 \Delta t) \Psi(t-\Delta t)+2 \Delta t \mathcal{L}(\Delta t) \mathcal{H}_{0} \Psi(t)
$$

where $\mathcal{L}(\Delta t)=\exp (\Delta t \mathcal{V})$. The amplification matrix, $\Psi(t+\Delta t)=\mathcal{G}_{\mathcal{L}}(\Delta t) \Psi(t)$, for the recurrence (5.9) satisfies the equation

$$
\mathcal{G}_{\mathcal{L}}(\Delta t)=\mathcal{L}(2 \Delta t) \mathcal{G}_{\mathcal{L}}^{-1}(\Delta t)+2 \Delta t \mathcal{L}(\Delta t) \mathcal{H}_{0}
$$

A deviation of the approximate solution $\mathcal{G}_{\mathcal{L}}^{n}(\Delta t) \Psi(0)$ from the exact solution relative to the energy norm is of order $\Delta t^{2}$ for any $n>0$. Thus, the scheme is convergent and, hence, a conditional stability exists for a sufficiently small $\Delta t>0$ according to a general theorem of Kantorovich [2] that establishes a general equivalence between convergence and conditional stability. The conditional stability of (5.9) can be understood from the following observation. Solving $(5.10)$ by perturbation theory in $\Delta t$, it is not hard to find that

$$
\mathcal{G}_{\mathcal{L}}(\Delta t)-\mathcal{G}_{V}(\Delta t)=\Delta t^{3} \mathcal{K}(\Delta t), \quad \mathcal{G}_{V}(\Delta t)=\mathcal{L}(\Delta t / 2) \mathcal{G}_{0}(\Delta t) \mathcal{L}(\Delta t / 2)
$$

where $\mathcal{K}(\Delta t)$ is regular in the vicinity of $\Delta t=0$ and vanishes whenever $\mathcal{H}_{0}$ and $\mathcal{V}$ commute, and $\mathcal{G}_{0}(\Delta t)$ is the amplification matrix when $\mathcal{V}$ is set to zero. The von Neumann condition is satisfied for $\mathcal{G}_{0}(\Delta t)$ for a sufficiently small $\Delta t>0$. Hence, powers of $\mathcal{G}_{0}(\Delta t)$ applied to $\Psi(0)$ cannot produce any exponential norm growth. Powers of $\mathcal{G}_{V}(\Delta t)$ differ from those of $\mathcal{G}_{0}(\Delta t)$ by factors that are powers of $e^{\Delta t \mathcal{V}}$ and, hence, can only produce exponential attenuation of the amplitude. Indeed, let $\Psi_{V}(t)=e^{t \mathcal{V}} \Psi(0)$. Then $\partial / \partial t\left(\Psi_{V}, \Psi_{V}\right)=2\left(\Psi_{V}, \mathcal{V} \Psi_{V}\right) \leq 0$ since $\mathcal{V}$ is negative semidefinite. Thus, the approximate solution produced by the amplification matrix $\mathcal{G}_{V}(\Delta t)$ has no exponential growth, while differing, relative to the energy norm, from that produced by $\mathcal{G}_{\mathcal{L}}(\Delta t)$ by order of $O\left(\Delta t^{2}\right)$. Therefore the modified leapfrog scheme can be made conditionally stable and as accurate as desired by reducing the time step.

It should be noted that our arguments do not prove that there cannot be any exponential growth of the norm $\|\Psi(t)\|=(\Psi(t), \Psi(t))^{1 / 2}$ in the modified leapfrog scheme (5.9). All we can claim is that $\|\Psi(t)\| \leq \exp (K t)$ which is also true for the conventional leapfrog scheme. The difference is that in the modified leapfrog scheme $K \sim O\left(\Delta t^{2}\right.$ ) as we have argued (a consequence of (5.11) and uniform boundedness of powers of $\mathcal{G}_{V}$ ), while in the conventional leapfrog scheme the constant $K$ is independent of $\Delta t$. Hence a possible exponential growth cannot be suppressed by reducing the time step in (5.3), while it can be done in (5.9).

\section{An example of the Lorentz model}

To illustrate our general method we give an example of the Lorentz model commonly used to describe dispersive media. In the field representation of the Hamiltonian for the Lorentz model, we make the following decomposition

$$
\mathcal{H}^{F}=\left(\begin{array}{cc}
\mathcal{H}_{0} & \mathcal{V}_{F M} \\
\mathcal{V}_{M F} & 0
\end{array}\right)+\left(\begin{array}{cc}
0 & 0 \\
0 & \mathcal{H}_{M}^{F}
\end{array}\right) \equiv \mathcal{H}_{0}^{F}+\mathcal{V}^{F}
$$


Substituting this decomposition into (5.9) we arrive at the following scheme

$$
\begin{aligned}
\psi^{F}(t+\Delta t) & =\psi^{F}(t-\Delta t)+2 \Delta t \mathcal{H}_{0} \psi^{F}(t)+2 \Delta t \sum_{a} \mathcal{V}_{F M a} \xi^{a}(t) \\
\xi^{a}(t+\Delta t) & =e^{2 \Delta t \mathcal{H}_{M a}^{F}} \xi^{a}(t-\Delta t)+2 \Delta t e^{\Delta t \mathcal{H}_{M a}^{F}} \mathcal{V}_{M F a} \psi^{F}(t) \\
e^{t \mathcal{H}_{M a}^{F}} & =e^{-\gamma_{a} t}\left[\cosh \tilde{\nu}_{a} t+\frac{\sinh \tilde{\nu}_{a} t}{\tilde{\nu}_{a}}\left(\mathcal{H}_{M a}^{F}+\gamma_{a}\right)\right]
\end{aligned}
$$

where $\tilde{\nu}_{a}=\left(\gamma_{a}^{2}-\omega_{a}^{2}\right)^{1 / 2}$ and the six-dimensional columns $\xi_{a}$ are introduced in Section 3. The exponential (5.15) is easy to compute by expanding $\mathcal{H}_{M a}^{F}$ in the Pauli matrix basis (a basis for the Lie algebra $s u(2))$ and then by using the well known formula for the exponential of a linear combination of Pauli matrices. For small attenuation, $\gamma_{a}<\omega_{a}$, we get $\tilde{\nu}_{a}=i \nu_{a}$. The hyperbolic functions in (5.15) become the trigonometric ones and $\tilde{\nu}_{a}$ is replaced by $\nu_{a}$. Eigenvalues of the matter Hamiltonian are $\lambda_{a}=-\gamma_{a} \pm \tilde{\nu}_{a}$. Hence, $\operatorname{Re} \lambda_{a}<0$ and amplitudes of the matter fields are always exponentially attenuated as $t \rightarrow \infty$, unless $\gamma_{a}=0$ leading to $\operatorname{Re} \lambda_{a}=0$.

The stability is ensured if $\mathcal{H}_{0}^{F}$ satisfies the von Neumann condition. Let $k_{\max }$ be the maximal norm of all wave vectors of the initial wave packet and $\omega_{p}^{\max }$ be the maximal value of $\omega_{p}=$ $\left(\sum_{a} \omega_{p a}^{2}\right)^{1 / 2}$ as a function of position, then a sufficient condition for stability reads

$$
\Delta t \sqrt{c^{2} k_{\max }^{2}+\left(\omega_{p}^{\max }\right)^{2}} \leq 1
$$

Here the idea of the frozen coefficients [17] has been used again. The left hand side of inequality (5.16) is nothing but the spectral radius of $\Delta t \mathcal{H}_{0}^{F}$ with frozen plasma frequencies so that $\omega_{p}=\omega_{p}^{\max }$. Note that it is not difficult to solve the characteristic equation for $\mathcal{H}_{0}^{F}$ with frozen plasma frequencies by using the Fourier basis. The scheme (5.9) becomes especially simple in the case of small attenuation $\gamma_{a}<\omega_{a}$. In the complex representation of the auxiliary fields (3.8) (cf. (3.7)) the matter Hamiltonians $\mathcal{H}_{M a}^{F}$ are diagonal and the action of its exponential is reduced to multiplication by a complex number $e^{i \nu_{a} \Delta t}$.

Finally, it should be mentioned that, by rearranging operators in the split, namely, by moving $\mathcal{V}_{F M}$ and $\mathcal{V}_{M F}$ to $\mathcal{V}^{F}$ in (5.12), the stability condition (5.16) can be weakened to $\Delta t c k_{\max } \leq 1$. This would come at the price of having a more complicated expression for $\mathcal{L}(\Delta t)$. In the case of the Lorentz model it can still be computed analytically. The new split can also be viewed as the use of the induction representation in the modified leapfrog scheme, $\mathcal{H}^{I}=\mathcal{H}_{0}^{I}+\mathcal{V}^{I}$ where $\mathcal{H}_{0}^{I}$ contains only the blocks of $\mathcal{H}^{I}$ with the $\boldsymbol{\nabla}$ operator. The proof of the weaker stability condition can be found in [14].

\section{Metal gratings in the Drude formalism}

Here we apply our method to gratings made of a metal whose optical properties are described by the Drude formalism. This is an actual numerical scheme used in simulations in Section 7. 
The metal dielectric constant as a function of frequency is given by

$$
\varepsilon(\omega)=1+\tilde{\chi}(\omega)=1-\frac{\omega_{p}^{2}}{\omega(\omega+i \eta)},
$$

where $\omega_{p}$ is the plasma frequency, which is zero in the vacuum and constant in the region occupied by the metal as shown in Fig.1, and $\eta$ is the absorption. The model coincides with a one-resonant Lorentz model if $\omega_{0}=0$ and $\eta \equiv 2 \gamma$. To satisfy the Gauss law exactly in simulations, we use the induction representation according to Section 5.II. An auxiliary field is chosen so that its first component equals $\mathbf{P}$ and the second is denoted $\boldsymbol{\xi}$. The Hamiltonian evolution equations are taken in the form

$$
\begin{aligned}
\dot{\mathbf{D}} & =c \boldsymbol{\nabla} \times \mathbf{B} \\
\dot{\mathbf{B}} & =-c \boldsymbol{\nabla} \times(\mathbf{D}-\mathbf{P}), \\
\dot{\mathbf{P}} & =\eta \boldsymbol{\xi} \\
\dot{\boldsymbol{\xi}} & =-\eta \boldsymbol{\xi}-\frac{\omega_{p}^{2}}{\eta}(\mathbf{D}-\mathbf{P}) .
\end{aligned}
$$

To apply the modified leapfrog scheme the Hamiltonian is split into the sum

$$
\begin{aligned}
\mathcal{H}^{I} & =\left(\begin{array}{cccc}
0 & c \boldsymbol{\nabla} \times & 0 & 0 \\
-c \boldsymbol{\nabla} \times & 0 & c \boldsymbol{\nabla} \times & 0 \\
0 & 0 & 0 & -\eta \\
-\omega_{p}^{2} \eta^{-1} & 0 & \omega_{p}^{2} \eta^{-1} & -\eta
\end{array}\right)=\mathcal{H}_{0}^{I}+\mathcal{V}^{I} \\
\mathcal{V}^{I} & =\operatorname{diag}(0,0,0,-\eta) .
\end{aligned}
$$

Clearly, $\mathcal{V}^{I}$ is negative semidefinite because $\eta>0$. The stability of the modified leapfrog scheme requires that eigenvalues of $\mathcal{H}_{0}^{I}$ have zero real parts. This is indeed the case. In the Fourier basis, the $\boldsymbol{\nabla}$ operator becomes $i \mathbf{k}$. It is straightforward to find the characteristic polynomial $\operatorname{det}\left(\mathcal{H}_{0}^{I}-\lambda\right)$. Its nonzero roots are $\lambda= \pm i \sqrt{c^{2} \mathbf{k}^{2}+\omega_{p}^{2}}$. Hence the scheme is stable if the time step is chosen so that the condition (5.16) is satisfied. In particular, $k_{\max }$ can be set to $k_{0}$ being the step of the reciprocal lattice and $\omega_{p}^{\max }$ is the plasma frequency of the metal (silver in our simulations, see Section 7). An explicit form of the modified leapfrog scheme for the split (6.1) reads

$$
\begin{aligned}
\mathbf{D}(t+\Delta t) & =\mathbf{D}(t-\Delta t)+2 c \Delta t \boldsymbol{\nabla} \times \mathbf{B}(t) \\
\mathbf{B}(t+\Delta t) & =\mathbf{B}(t-\Delta t)-2 c \Delta t \boldsymbol{\nabla} \times[\mathbf{D}(t)-\mathbf{P}(t)] \\
\mathbf{P}(t+\Delta t) & =\mathbf{P}(t-\Delta t)-2 \eta \Delta t \boldsymbol{\xi}(t) \\
\boldsymbol{\xi}(t+\Delta t) & =e^{-2 \eta \Delta t} \boldsymbol{\xi}(t-\Delta t)-2 \Delta t \omega_{p}^{2} \eta^{-1} e^{-\eta \Delta t}[\mathbf{D}(t)-\mathbf{P}(t)] .
\end{aligned}
$$

The action of the curl is computed by the fast Fourier method in combination with a change of variables that enhances the sampling efficiency near the metal-vacuum interface. The details are in Section 7. 


\section{$7 \quad$ Results for extraordinary transmission gratings}

To test our method we applied it to study transmission properties of metal and dielectric gratings suspended in vacuum. Transmission and reflection gratings have been subject of a number of experimental and theoretical works [9]-[12], [18]. The interest is stimulated by nearly $100 \%$ transmission or reflection within narrow wavelength range with possibility of using such grating as efficient filters. Moreover, extraordinary optical transmission has been observed in the $2 D$ hole arrays [8], further stimulating theoretical and experimental interest to transmission properties of nanostructured materials [13].

In Fig.1 we schematically represent our system comprising a metallic or dielectric slab with gratings. $D$ is a grating period, $a$ the size of the grating and $h$ its thickness. For the sake of comparison with previously published results we have chosen $D=1.75 \mu \mathrm{m}$ and $a=0.30 \mu \mathrm{m}$. Transmission and reflection coefficients are computed as a function of the grating thickness $h$. An incident electromagnetic wave packet propagates along the $z$-direction, normal to the slab. The polarization is such that the electric field vector is perpendicular to the gratings, while the magnetic field is parallel to them (the so called $p$-polarization). Calculations are performed in a finite $(x, z)$ box of the size $-15 D<z<12 D,-D / 2<x<D / 2$. A uniform mesh of typically 256 knots is used in the $x$-direction and a nonuniform mesh of 512 knots generated by the change of variables is used in the $z$-direction. The change of variables is used to enhance the sampling efficiency near the two interfaces in the $z$-direction. Periodic boundary conditions are insured in $x$ through the pseudospectral approach based on the Fast Fourier Transform. To suppress an artificial reflection of the wave packet, absorbing layers are introduced at the box boundaries $z= \pm 15 \mathrm{D}$. The initial wave packet is Gaussian, which allows us to obtain transmission (reflection) coefficients within the entire frequency bandwidth of the initial wave packet by a single simulation. In what follows we are mainly interested in transmission (reflection) of the radiation with wavelengths larger than the grating period (zero order diffraction). Thus, reflected or transmitted waves propagate in the direction of the $z$-axis, the same as the incident radiation. Note, however, that this is not a limitation of our method which allows for a priori extraction of the entire scattering matrix for all wave vectors.

In Fig.2 we show transmission and reflection coefficients obtained for metallic gratings of variable depth. The dielectric response of the metal is described within the Drude formalism (see Section 6). Following [10] we use $\omega_{p}=9 \mathrm{eV}$ and $\eta=0.1 \mathrm{eV}$ representative for silver. As clearly seen in the Figure, the transmission coefficients exhibit narrow resonances for certain wavelengths. With increasing thickness of the gratings, $h$, the number of resonant structures increases. Our results are in a full agreement with previously published theoretical studies [10]. The only difference being, that the model used in [10] assumes perfect metal surfaces inside the gratings, neglecting possible absorption of the radiation. This leads to $100 \%$ transmission at the resonant frequencies. In our case, a part of radiation is absorbed by the metal so that the transmission never reaches $100 \%$.

The observed structures in transmission are associated with resonant modes of the elec- 
tromagnetic field produced by coupled surface electromagnetic modes (called surface plasmon polaritons) and waveguide modes inside the gratings. Some of these resonances posses relatively long lifetimes. This can be immediately inferred from their width, as, e.g., the resonance located at $\lambda=1.1 D$ in the case of the grating with thickness $h=1.4 \mu m$. Another way to observe the trapped field resonances is to look at the time dependence of the field transmitted in the $z$-direction. The signal in Fig. 3 is registered by a detector placed at the distance of $3.5 \mathrm{D}$ behind the gratings. As seen in the figure, as soon as the resonances are populated by an incident $25 \mathrm{fs}$-long pulse, they radiate the field during at least $125 \mathrm{fs}$. Here the radiation time is determined by the lifetime of the narrowest resonance located at $\lambda=1.1 D$. It is worth mentioning that, as shows the sum of the transmission and reflection coefficients, the total absorption is largest at the resonance positions, i.e., when the interaction time between the radiation (trapped mode) and the metal is large.

Finally, Fig. 4 shows the field structure of a trapped mode corresponding to the narrowest resonance observed with the $h=2.4 \mu \mathrm{m}$ thickness grating. The $E_{x}$ component of the electric field is presented. It is obtained via sufficiently long time propagation so that contributions from less long-lived states vanish.

In Figs. 5 and 6 we show reflection coefficients for dielectric gratings suspended in vacuum. Simulations are performed using the conventional leapfrog scheme (5.3) applied to (4.5). Since the attenuation is absent, the scheme is stable if the time step satisfies (5.6). The dielectric material is modeled through the frequency independent dielectric constant $\varepsilon=2$ (Fig.5) and $\varepsilon=4$ (Fig.6). Only the reflection coefficient is shown here since there is no absorption of the radiation in dielectric so that the transmission can simply be inferred from the unitarity of the scattering matrix. Without gratings the dielectric slabs are basically transparent in both cases. Introducing grating structures results in a complete reflection of the incident radiation within an extremely narrow wavelength bandwidth. The associated guided mode resonances have been extensively discussed in the literature [18]. In the case of $\varepsilon=2$ the resonances are so narrow that extraction of frequency dependent transmission and reflection coefficients by the Fourier transform of the scattered wave would require too large propagation time (see also Fig. 7). We had to stop our wave packet propagation before the radiation emitted by resonances ended. This explains why the reflection coefficients do not reach its maximal value 1 in this case. Consistently with the metal case, the number of resonant structures increases with increase of the width $h$ of the gratings. The width of resonances increases with $\varepsilon$ as follows from the comparison of Figs. 5 and 6 . Note also that resonances are associated with Fano profiles that usually arise because of the interference between the non-resonant and resonant contributions to the scattered wave. Such narrow reflection structures in the case of dielectric gratings have been usually studied by stationary methods in the frequency domain. An important advantage of the time-dependent study is that one has an immediate access to all the details the temporal evolution of electromagnetic fields in any desired part of the system.

In Fig. 7 we show the time dependence of the field transmitted in the $z$-direction for gratings characterized by $\varepsilon=2$ and $h=0.8 \mu \mathrm{m}$. With these parameters there is only one resonance in the reflection spectrum. The signal is registered by a detector placed at the distance of $3.5 \mathrm{D}$ 
behind the gratings. First, we observe that a $25 \mathrm{fs}$ incident pulse is transmitted through the structure without modification. The lasing effect, when the transmitted field is followed by basically monochromatic radiation, can clearly be seen. For readability reasons we could not show the complete time evolution in the figure, but the lasing effect lasts for at least (!) $2 p s$ reflecting an extraordinary long lifetime of the trapped resonant field. It is this radiation which comes in the phase opposite to the corresponding harmonic in the transmitted initial pulse and leads, finally, to the zero transmission at the corresponding frequency. The same lasing effect to the left from the grating structure is responsible for the $100 \%$ reflection at the same frequency.

In Fig. 8 we show a typical structure of the field corresponding to a trapped (resonant) mode. The $D_{x}$ component of the electric induction is represented in the case of dielectric gratings with $\varepsilon=4$. The thickness of the dielectric slab is $h=0.6 \mu \mathrm{m}$. Note that in contrast to the metal grating structure, the field in the present case occupies the entire slab and not only the vacuum part of the grating.

\section{Conclusions}

We have developed a time domain algorithm for the initial value problem for the Maxwell's theory of linear passive media. The algorithm is based on $(i)$ the Hamiltonian formalism for evolution differential equations, $(i i)$ the Fourier pseudospectral method in which the sampling efficiency in designated space regions is enhanced by a suitable change of variables, and (iii) the modified leapfrog scheme. We have analyzed the stability of the algorithm and found explicit stability conditions when passive media are described by multi-resonant Lorentz models. We have implemented and tested our algorithm for extraordinary transmission and reflection gratings whose optical properties have been studied in a number of theoretical and experimental works. Numerical simulations based on our algorithm are shown to produce extremely accurate data for the well studied far-field (zero-order diffraction) at relatively low computational costs. A single simulation of an incident wideband wave packet is sufficient to determine transmission and reflection properties of the gratings in the frequency range of the initial wave packet. In addition, our algorithm allows us to see a real time dynamics of formation of long-living resonant excitations of electromagnetic fields in the grating as well as formation of transmitted and/or reflected wave fronts in the entire frequency range of the initial wave packet. It is believed that our algorithm would be useful for numerical studies of other nanostructured materials.

\section{Acknowledgments}

S.V.S thanks the Laboratory of Atomic and Molecular Collisions of the University of ParisSud for the warm hospitality and Dr. R. Albanese (Brooks, Air Force Base, TX) for his continued interest and support of this project. The work of S.V.S. has been supported in part by US Air Force Grants F4920-03-10414 and F49620-01-1-0473. 


\section{References}

[1] V.I. Arnold, Mathematical methods of classical mechanics, Springer-Verlag, Berlin, 1989.

[2] R. Richtmyer and K.W. Morton, Difference Methods for Initial Value Problems, Interscience Publishers, New York, 1967.

[3] M. Pickering, An introduction to fast Fourier transform methods for partial differential equations, Research Study Press, John Wiley \& Sons Inc., New York, 1986;

E.O. Brigham, The fast Fourier transform and applications, Prentice-Hall, Inc., Eglewood Cliffs, New Jersey, 1988.

[4] E. Fattal, R. Baer and R. Kosloff, Phys. Rev. E 53 (1996) 1217;

D. Lemoine, Chem. Phys. Lett. 320 (2000) 492.

[5] A.G. Borisov, J. Chem. Phys. 114, 7770 (2001).

[6] L.D. Landau and E.M. Lifshitz, Electrodynamics of continuous media, Theoretical Physics, Vol. VIII, Oxford, Pergamon, New York, 1984.

[7] B. Fornberg, A practical guide to pseudospectral methods, Cambridge University Press, Cambridge, 1996;

J.P. Boyd, Chebyshev and Fourier spectral methods, Springer-Verlag, New York, 1989.

[8] T.W. Ebbesen, H.J. Lezec, H.F. Ghaemi, T. Thio, and P.A. Wolff, Nature (London) 391, 667 (1998).

[9] M.M.J. Treacy, Appl. Phys. Lett. 75, 606 (1999);

M.M.J. Treacy, Phys. Rev. B 66, 195105 (2002).

[10] F.J. Garcia-Vidal, L. Martin-Moreno, Phys. Rev. B66, 155412 (2002);

U. Schröter and D. Heitmann, Phys. Rev. B 58, 15419 (1998).

[11] J.A. Porto, F.J. Garcia-Vidal, and J.B. Pendry, Phys. Rev. Lett. 83, 2845 (1999);

Ph. Lalanne, J.P. Hugonin, S. Astilean, M. Palamaru, and K.D. Möller, J. Opt. A: Pure Appl. Opt. 2, 48 (2000).

[12] J.M. Steele, C.E. Moran, A. Lee, C.M. Aguirre, and N.J. Halas, Phys. Rev. B 68, 205103 (2003).

[13] H.J. Lezec, A. Degiron, E. Devaux, R.A. Linke, L. Martin-Moreno, F.J. Garcia-Vidal, T.W. Ebbesen, Science 297, 820 (2002);

L. Martin-Moreno, F.J. Garcia-Vidal, H.J. Lezec, A. Degiron, and T.W. Ebbesen, Phys. Rev. Lett. 90, 167401 (2003);

S. A. Darmanyan and A. V. Zayats, Phys. Rev. B 67, 035424 (2003);

A.P. Hibbins, J.R. Sambles, and C.R. Lawrence, Phys. Rev. E 61, 5900 (2000);

F. Yang and J.R. Sambles, Phys. Rev. Lett. 89, 063901 (2002);

W.-C. Tan, T.W. Preist, and R.J. Sambles, Phys. Rev. B 62, 11134 (2000) 
[14] S.V. Shabanov, Electromagnetic pulse propagation in passive media by path integral methods, a LANL e-preprint, 2003, math.NA/0312296 (submitted to Appl. Comput. Harm. Analysis)

[15] R.M. Joseph, S.C. Hagness, and A. Taflove, Opt. Lett. 16 (1991) 1412;

L.Gilles, S.C. Hagness, and L. Vázquez, J. Comput. Phys. 161 (2000) 379.

[16] A.G. Borisov and S.V. Shabanov, Chem. Phys. Lett. 361 (2002) 15.

[17] H. Shintani and K. Tomoeda, Hiroshima Math. J., 7 (1977) 309.

[18] K. Koshino, Phys. Rev. B 67, 165213 (2003);

L. Pilozzi, A. D’Andera, R. Del Sole, Phys. Rev. B 54, 10763 (1996) 


\section{Figures}

Fig. 1 A schematic representation of the studied system. The incident wave packet propagates along the normal to the slab containing the gratings (z-direction).

Fig. 2. Calculated zero-order transmission (solid lines) and reflection (dashed lines) coefficients for metallic gratings described in the text. The results are presented as a function of the wavelength $\lambda$ of the incident radiation measured in the units of the period of the gratings, $D$. Different panels of the figure correspond to the different thickness $h$ of the gratings, as indicated.

Fig. 3. The electric field measured by a detector placed behind the metallic gratings with thickness $h=1.4 \mu \mathrm{m}$. Only the field corresponding to the zero-order transmitted wave propagating along the $z$-axis is represented. It is obtained by the Fourier analysis of the $x$ coordinate dependence of the field at the detector position. The signal is shown as a function of time measured in femtoseconds.

Fig. 4. A snapshot of the $x$-component of the electric field, $E_{x}$ for the grating thickness $h=2.4 \mu \mathrm{m}$. The results are presented as a function of $x$ and $z$ coordinates measured in units of $D$. Red and blue colors correspond, respectively, to positive and negative values of the field. The snapshot has been produced after a sufficiently long propagation time so that the field pictured in it indeed corresponds to the long-lived resonance giving enhanced transmission at $\lambda=1.15 D$ (see Fig.2).

Fig. 5. Calculated zero-order reflection coefficient for dielectric gratings with $\varepsilon=2$. The results are presented as a function of the wavelength $\lambda$ of the incident radiation measured in the units of the period of the gratings, $D$. Different panels of the figure correspond to the different thickness $h$ of the gratings, as indicated.

Fig. 6. Calculated zero-order reflection coefficient for dielectric gratings with $\varepsilon=4$. Results are presented as a function of the wavelength $\lambda$ of the incident radiation measured in the units of the period of the gratings, $D$. Different panels of the figure correspond to the different thickness $h$ of the gratings, as indicated.

Fig. 7. The electric field measured by a detector placed behind the dielectric grating with thickness $h=0.8 \mu \mathrm{m}$ and dielectric constant $\varepsilon=4$. Only the field corresponding to the zero-order transmitted wave propagating along the $z$-axis is represented. It is obtained by the Fourier analysis of the $x$-coordinate dependence of the field at the detector position. The signal is shown as a function of time measured in femtoseconds.

Fig. 8. A snapshot of the $x$-component of the electric induction, $D_{x}$ for the dielectric grating with dielectric constant $\varepsilon=4$ and thickness $h=0.6 \mu \mathrm{m}$. The results are presented as a function of $x$ and $z$ coordinates measured in units of $D$. Red and blue colors correspond, respectively, to positive and negative values of the induction. The snapshot has been produced after a sufficiently long propagation time so that the induction pictured in it indeed corresponds 
to the resonance giving enhanced transmission at $\lambda \cong 1.3 D$ (see Fig.6). 
This figure "Fig1.png" is available in "png" format from: http://arxiv.org/ps/physics/0312103v1 
This figure "Fig2.png" is available in "png" format from: http://arxiv.org/ps/physics/0312103v1 
This figure "Fig3.png" is available in "png" format from: http://arxiv.org/ps/physics/0312103v1 
This figure "Fig4.png" is available in "png" format from: http://arxiv.org/ps/physics/0312103v1 
This figure "Fig5.png" is available in "png" format from: http://arxiv.org/ps/physics/0312103v1 
This figure "Fig6.png" is available in "png" format from: http://arxiv.org/ps/physics/0312103v1 
This figure "Fig7.png" is available in "png" format from: http://arxiv.org/ps/physics/0312103v1 
This figure "Fig8.png" is available in "png" format from: http://arxiv.org/ps/physics/0312103v1 